years, quality-adjusted life-years, and incremental cost-effectiveness ratios. Univariate and probabilistic sensitivity analyses were performed.

Results Three scenarios were simulated in which the proportion of patients referred to thrombectomy after 6 hours was varied from $5 \%$, to $10 \%$ and to $15 \%$ with SPCCT while it remained constant to $0 \%$ with CT-CTA. Three levels of SPCCT accuracy were simulated (perfect positive predictive value (PPV) (100\%), intermediate PPV (78.1\%), and low PPV $(46.9 \%))$. Over the three-month and lifetime horizons, all SPCCT-based scenarios improved QALYs and LYs compared to CT-CTA. Based on the currently used estimates, the probability that SPCCT is cost-effective at a willingness-to-pay threshold of $£ 10000$ per QALY is above 50\% in all scenarios.

Conclusions Based on preliminary results, replacing CT-CTA with SPCCT to identify and treat ischaemic stroke patients beyond 6 hours may be good value for money. However, additional data regarding clinical outcomes following SPCCT based strategies are needed before the cost-effectiveness can be fully assessed.

\section{AUDIT OF A NEWLY DEVELOPED AMBULATORY CARE SERVICE AT DIANA PRINCESS OF WALES HOSPITAL GRIMSBY UK}

Asem Ali, Jaidev Karmani. Northern Lincolnshire and Goole NHS Foundation Trust, UK

\subsection{6/postgradmedj-2018-fpm.24}

Introduction The aim of the ambulatory care unit is to enable patients to be assessed, observed, diagnosed and treated without the need to be admitted to hospital and meeting their clinical needs by greater consistency of care through reduced clinical variability and improved patient experience, which can potentially reduce the number of clinical admissions, reduce time spent in hospital and helping to reduce overcrowding in the Emergency Department. We looked at performance of newly developed ambulatory care service at Diana Princess of Wales Hospital over 30 days period.

Methods Ambulatory care patients were selected who attended the service from 1 st of October 2017 to 30th of October 2017. Information was collected by looking at the ambulatory care record spreadsheet, medical notes, discharge letters and hospital ambulatory care data. Service impact was analysed by numbers of service consumers, source of referral, impact on Accident and Emergency four hours target, appropriateness of referral, zero day discharges, length of stay for inpatients and diagnosis at discharge.

Results Patients were admitted to ambulatory care using the AMBS criteria and different ambulatory care models. Over the 30 day period, 260 attendances were recorded (male 123, female 137) which was $20 \%$ of the total medical admissions. $8 \%$ of the referrals were from the Accident and Emergency Department. The majority of patient were referred as chest pain and shortness of breath, the most common diagnosis being musculoskeletal chest pain. Other diagnoses included cellulitis, pulmonary embolism, respiratory tract infection, angina and urinary tract infection. More than $92 \%$ of the patients were discharged same day. Only $8 \%$ of the patients were admitted to the Acute Admission Unit and among these 50\% of the patients were referred inappropriately. $16 \%$ of patients were classed as re-attendees, mainly for treatment with iv antibiotics for cellulitis or pending next day investigation. The percentage of zero day discharges increased to $15 \%$ and there was an improvement in the Accident and Emergency four hour target exceeded to $5 \%$.

Conclusion The newly developed ambulatory care service has helped to increase zero day discharges and improved the Accident and Emergency four hour target. Ambulatory care has not been utilised by primary care colleagues. Audit Results were shared with CCG to improve the referral rate by Primary Care. More investment is needed in future in ambulatory care by reinvesting money saved by reduced length of stay.

\section{TESTICULAR SEMINOMA IN AN INFERTILE ADULT PATIENT WITH PERSISTENT MULLERIAN DUCT SYNDROME}

${ }^{1}$ Shaden Saleh Al Mousa, ${ }^{1}$ Nufe Al Mansor, 'Ammar Ashraf, ${ }^{1}$ Mohamed Tahar Yacoubi, ${ }^{1}$ Peter Sylivanus Bahame, ${ }^{2}$ Sohail Iqbal, ${ }^{3}$ Usman Saeed. ' King Abdulaziz Hospital, Ministry of National Guard Health Affairs, Al-Ahsa, Kingdom of Saudi Arabia; ${ }^{2}$ Wythenshawe Hospital, Manchester University NHS Foundation Trust; ${ }^{3}$ Royal Blackburn Hospital, UK

\subsection{6/postgradmedj-2018-fpm.25}

Introduction Persistent Mullerian duct syndrome (PMDS) is an autosomal recessive disorder characterized by the persistence of Mullerian duct derivatives (uterus, cervix, fallopian tubes and proximal vagina) in a phenotypically and karyotypically male patient. This internal male pseudohermaphroditism is usually an incidental diagnosis in children who present with cryptorchidism and inguinal hernia or in adults with cryptorchidism, infertility or an abdominal mass. PMDS is associated with a higher risk of malignant transformation of gonads. Here we present a case of testicular seminoma in an adult infertile patient with persistent MDS. The patient had laparoscopic bilateral orchidectomy with removal of fallopian tubes and commenced on long-term testosterone supplement therapy.

Subjects and materials A 42 years old morbidly obese (body mass index 57) infertile male patient had an elective admission for bariatric surgery. During pre-operative assessment, he was investigated for infertility and had an inguinoscrotal ultrasound to locate undescended testes. Postoperatively, he had MRI abdomen and pelvis. Six months later he had an urgent contrast enhanced CT abdomen and pelvis to rule out the causes of his right flank pain.

Result Pre-operative inguinoscrotal ultrasound revealed no testicular tissue in either the inguinal canals or the scrotum [figure 1]. Genetic screening confirmed $46 \mathrm{XY}$ Karyotyping and hormonal profile showed raised FSH (26.3 IU/L) and $\mathrm{LH}$ (14.2 IU/L) and normal testosterone level $(11.3 \mathrm{ng} / \mathrm{ml})$. MRI pelvis confirmed the presence of a small, rudimentary uterus and bilateral fallopian tubes and identified bilateral adnexal masses (gonads) measuring about $3 \times 2 \mathrm{~cm}$. Emergency contrast enhanced CT abdomen [figure 1] showed a complex heterogeneous mass containing some air foci in right iliac fossa with twisted engorged vascular pedicle unrelated to bowel loops, presumed to be a twisted gonad. Following imaging evaluation, patient underwent laparoscopic removal of this mass, mildly congested appendix, normal looking left gonad and both fallopian tubes. Rudimentary uterus was left behind undisturbed. Histopathological analysis confirmed these gonads to be testicles with marked atrophic changes. Right sided testicle showed haemorrhagic infarction consistent with torsion along with a $5.5 \mathrm{~cm}$ classic seminoma [figure 1]. Full 


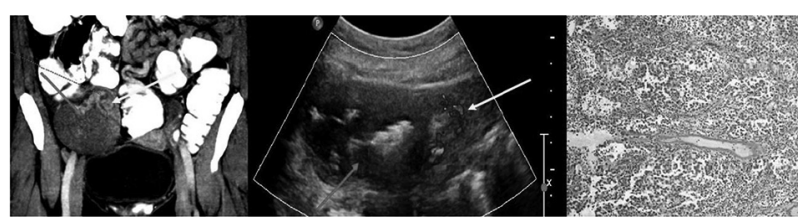

Abstract 14 Figure 1 Testicular Seminoma - From left to right coronal reconstruction of CT scan and US images depicting RIF mass in red arrow and twisted pedicle in yellow arrow. Histopathology image demonstrate malignant cell proliferation of classical seminomatous noncohesive, large, round \& atypical cells with atypical nuclei owing prominent nucleoli \& granular cytoplasm in right gonad

circumference fallopian tube and fimbria were identified bilaterally. Because of bilateral orchidectomy with normal male karyotype and normal male secondary sexual characters, regular testosterone hormone replacement therapy (testosterone $1000 \mathrm{mg} /$ every 3 month) was instituted.

Conclusion Patients with persistent Mullerian duct syndrome or internal male pseudohermaphroditism have 46 XY karyotype, near normal development of secondary sexual characters and persistent Mullerian duct structures i.e., uterus, cervix, fallopian tubes and proximal vagina. Our patient was diagnosed very late possibly due to cultural issues and stigmata attached to pseudohermaphroditism.

\section{OVARIAN GERM CELL TUMOURS; EXPERIENCE OF A REGIONAL CENTRE}

${ }^{1}$ Douglas Cartwright, ${ }^{2}$ Ashita Waterston, ${ }^{2}$ Jeff White. ${ }^{1}$ NHS Greater Glasgow and Clyde; ${ }^{2}$ Beatson West of Scotland Cancer Centre

\subsection{6/postgradmedj-2018-fpm.26}

Introduction Ovarian germ cell tumours are rare but aggressive cancers which have histological and treatment similarities with the more common male germ cell tumours. However the outcomes are poorer for women with germ cell tumours than for men. ${ }^{1}$ Historically, in the west of Scotland, female germ cells patients were managed within standard gynaecological cancer services and male patients in a dedicated germ cell unit. Due to the highly specialised nature of the condition and the experience of the clinicians using similar chemotherapy regimens, the management of ovarian germ cells was incorporated into the germ cell unit. We present the experiences of this unit over the last 6 years.

Method We reviewed data from all women with ovarian germ cell tumours treated or followed up under the regional germ cell unit. Fertility is an important consideration for these young women and we investigated the rate of fertility sparing surgery, utilisation of pre-chemotherapy fertility treatments and the rate of successful pregnancies. With the Introduction of the Cisplatin, Vincristine, Methotrexate, Bleomycin with alternate Actinomycin, Cyclophosphamide and Etoposide chemotherapy regimen (POMB/ACE) there was a concern that this will cause problems with monitoring during treatment as it associated with elevation of alpha fetoprotein (AFP) levels which is used as a marker or response. We compared AFP levels between $\mathrm{POMB} / \mathrm{ACE}$ and the bleomycin, etoposide, cisplatin (BEP) regimen used in the adjuvant or neo-adjuvant setting.

Results 26 patients have been treated or followed up by the unit over the last 6 years. Median follow up is 55 months (range 3 months - 31 years). The all-cause mortality is 0 and the relapse rate is $19 \%$. Relapses were spread equally through the treatment groups. 21 out of 24 eligible patients received fertility sparing surgery. Of these there has been 6 successful pregnancies following treatment.

There was no significant difference in AFP levels between BEP and POMB/ACE groups.

Conclusions Outcomes for ovarian germ cell patients in the west of Scotland are comparable to published case series. The low incidence makes it difficult to identify statistically significant differences however we hope to promote collaboration and gather more data through a national ovarian germ cell database set up through the Scottish Germ Cell Group.

\section{REFERENCE}

1. Murugaesu N, Schmid P, Dancey G, Agarwal R, Holden L, McNeish I, et al. Malignant ovarian germ cell tumors: Identification of novel prognostic markers and long-term outcome after multimodality treatment. I Clin Oncol 2006;24:4862-6.

\section{A CASE OF PYREXIA OF UNKNOWN ORIGIN AND RECURRENT HOSPITAL ADMISSIONS IN A CARDIAC PATIENT}

${ }^{1}$ Syed Emir Irfan Wafa, ${ }^{2}$ Raheel Ahmed, ${ }^{3}$ Kay Teck Ling, ${ }^{3}$ Peter Carey. ${ }^{1}$ University Hospitals of Leicester NHS Trust; ${ }^{2}$ University Hospitals of North Midlands NHS Trust; ${ }^{3}$ University Hospitals of Derby and Burton NHS Foundation Trust

\subsection{6/postgradmedj-2018-fpm.27}

Introduction Pyrexia of unknown origin (PUO) is defined as a temperature greater than $38.3^{\circ} \mathrm{C}$ on several occasions, accompanied by three weeks of illness and a failure to reach a diagnosis after one week of inpatient investigations. ${ }^{1}$ We describe a case of a 72-year-old man with a significant cardiac history and a pacemaker in-situ presented to the emergency department of a district general hospital with sepsis five days after he had his pacemaker unit batteries changed. He had deranged vital signs, productive cough and pyrexia. He had a normal chest plain radiograph (CXR). However, with right basal crackles on auscultation, he was empirically treated for a lower respiratory tract infection (LRTI) with intravenous antibiotics. The patient was discharged but had to be readmitted four times over the span of 4 months due to recurrent pyrexia. Repeated Trans-thoracic (TTE) and Trans-oesophageal (TOE) echocardiograms, and CT neck/thorax/abdomen/pelvis were done however no evidence of infection was found on the scans. A FDG-PET scan [figure 1] was eventually done which showed evidence of pacemaker-lead infection. His pacemaker unit was removed and replaced with a REVEAL device. He improved clinically with no further hospital admission to date.

Methods Routine blood test only showed raised WCC and CRP. Connective tissue disease screen, viral and tumour
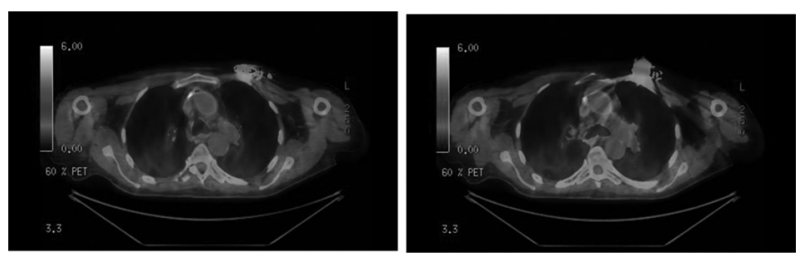

Abstract 16 Figure 1 FDG-PET scan images showing increased focal activity around pacemaker leads 